\title{
Resiliência regional: um estudo bibliométrico a partir da Web of Science
}

\author{
Regional resilience: a bibliometric study from the Web of Science \\ Newton da Silva Miranda Júnior ${ }^{1}$ \\ Orcid: https://orcid.org/0000-0002-2876-4016 \\ Valmir Emil Hoffmann ${ }^{2}$ \\ Orcid: https://orcid.org/0000-0002-8977-8454
}

\begin{abstract}
Resumo
Este estudo apresenta os principais tópicos e subtópicos da literatura acerca resiliência sob enfoque regional. Para esse fim, recorreu-se à rede de cocitações criada pelo programa de cientometria CiteSpace a partir da base integral de 829 artigos extraídos da Web of Science. Com base no método bibliométrico e delimitado a 61 artigos inseridos na área de conhecimento business economics, publicados no período de 2010 a 2017, o estudo apresenta também a demografia da produção teórica e empírica. Em adição, com base nos 50 artigos empíricos dessa área de conhecimento traçada, discute-se a tipificação metodológica e listam-se os fatores e variáveis comumente associados na literatura com a resiliência de regiões. Uma vez que tratar de resiliência implica, em muitos casos, assumir a ocorrência de um choque, constatou-se que os estudos voltados ao âmbito regional de resiliência referiram-se, majoritariamente, à crise econômico-financeira mundial de 2008-2009.
\end{abstract}

Palavras-chave: Resiliência Regional. Resiliência Regional Econômica. Bibliometria.

\begin{abstract}
This study presents the main topics and subtopics of the literature on resilience under a regional approach. A cocitation network was created by the CiteSpace scientometry program from the integral base, formed by 829 articles, indexed in the Web of Science. The study also presents the demographics of theoretical and empirical production, published in the period from 2010 to 2017 , through the bibliometric method and based on the 61 articles belonging to the business economics knowledge area. In addition: methodological typification is discussed based on theoretical and empirical articles found in this area of knowledge; and the factors and variables commonly associated by empirical articles with the resilience of regions are listed. Since dealing with resilience implies, in many cases, assuming the occurrence of a shock, the results found in this study indicate that studies focused on the regional scope of resilience mainly referred to the 2008-2009 global economic and financial crisis.
\end{abstract}

Keywords: Regional Resilience. Regional Economic Resilience. Bibliometric study.

\footnotetext{
${ }^{1}$ Universidade de Brasília (UnB), Brasil. E-mail: newtondasmjr@gmail.com

${ }^{2}$ Universidade de Brasília (UnB), Brasil. E-mail: ehoffmann@unb.br
} 


\section{INTRODUÇÃOO}

A ideia de resiliência não é uma novidade. A concepção originária desse termo provém dos estudos das ciências de materiais voltados às propriedades elásticas de corpos (MARTIN-BREEN; ANDERIES, 2011) que possibilitam a eles o armazenamento de energia de deformação sem, contudo, implicar em danos ou colapsos em suas estruturas (GORDON, 1978). Em paralelo à disseminação do pensamento sistêmico - em meados da década de 1960, o termo resiliência foi inserido no campo das ciências naturais e, por conseguinte, novas concepções do termo surgiram (DAVOUDI et al., 2012).

Sob a perspectiva de sistemas ecológicos, Holling (1973), definiu resiliência como as capacidades de sistemas de absorver alterações e, mesmo assim, persistir. No início da década de 1990, autores como Masten, Best e Garmezy (1990, p.426) abordaram a resiliência em nível de indivíduos sob a concepção de "processo, capacidade ou resultado de uma adaptação bem-sucedida, apesar das circunstâncias desafiadoras ou ameaçadoras". Em meados da década de 1990, a resiliência também passou a ser utilizada no sentido de capacidade ou habilidade de ajustamento de comunidades a estresses prologados (BROWN; KULIG, 1996).

Além desses campos, a resiliência adentrou, sobremodo ao longo da década de 2010, áreas voltadas ao estudo do espaço geográfico sob o entendimento de que ele também pode apresentar comportamento resiliente frente a perturbações (CELLINI; CUCCIA, 2015). Sobremodo após um dos maiores choques econômicos desde o último século, a crise financeira mundial de 2008-2009 (DUBÉ; POLĖSE, 2015), a geografia econômica tem investigado fatores relacionados ao território como fonte de resiliência de regiões (BOSCHMA; MARTIN, 2010), uma vez que essa crise mundial expôs a vulnerabilidade de regiões (DAVIES, 2011).

Nessa perspectiva, a resiliência na dimensão regional pode ser entendida como a capacidade de resistência ou de recuperação de uma economia frente a distúrbios que impactam a trajetória de crescimento e/ou de desenvolvimento de uma região (MARTIN; SUNLEY, 2015). Em geral, crises econômico-financeiras são comumente o cerne dos estudos inseridos na dimensão regional de resiliência.

Sabendo-se que as revisões de estudos anteriores de uma área de conhecimento contribuem ao avanço dela por sinalizarem o processo de evolução de seus conceitos e teorias (LI; MA; QU, 2017), este estudo objetivou identificar os principais tópicos e subtópicos de discussão presentes na literatura de resiliência regional e verificar a evolução deles ao longo do tempo. O programa de cientometria CiteSpace foi utilizado para essas finalidades a partir de 829 artigos, publicados no período de 1993 a 2017, extraídos da base acadêmica $W e b$ of Science (WoS).

Em complemento, a área business economics da WoS foi selecionada no intuito de se realizar revisão bibliométrica acerca da literatura de resiliência regional econômica. Essa seleção retornou 61 artigos teóricos e empíricos publicados no período de 2010 a 2017 em periódicos das áreas de economia, administração e geografia. Nesta etapa do estudo, foram investigadas as métricas de demografia da produção, tais como: distribuição temporal, artigos mais citados, a produtividade por autores e por periódicos e seus respectivos fatores de impacto.

$\mathrm{Na}$ segunda etapa da revisão bibliométrica, realizada com os 50 artigos empíricos dentre aqueles encontrados, o objetivo foi verificar as métricas acerca da tipificação metodológica da produção científica empírica, tais como: abordagem de pesquisa, tipo, recorte e formas de coleta 
de dados, técnicas analíticas, regiões abordadas e finalidade dos estudos ao longo do tempo. Ademais, dada a lacuna de estudos acerca dos determinantes da resiliência regional, conforme apontado por Martin e Sunley (2015), buscou-se identificar - com base no modelo desses autores - os fatores associados pela literatura empírica à capacidade resiliente de regiões.

$\mathrm{O}$ artigo encontra-se estruturado da seguinte maneira: o referencial teórico discute brevemente as três principais correntes sobre resiliência (de engenharia, sistema ecológico e evolutiva) no intuito de situar o leitor quanto à discussão presente na literatura. $\mathrm{Na}$ metodologia são informados os procedimentos para a realização da cientometria e os critérios utilizados para seleção de artigos da revisão bibliométrica. Nos resultados, além da rede de cocitações e das análises bibliométricas, destacam-se os determinantes de resiliência regional econômica. Por fim, nas conclusões são realizadas reflexões a partir dos achados e descritas as contribuições do estudo.

\section{AS PRINCIPAIS PERSPECTIVAS DE ANÁLISE ACERCA RESILIÊNCIA REGIONAL}

O entendimento de resiliência é moldado por concepções que perpassam diversos campos de conhecimento e, como reflexo, diferentes pontos de referência são adotados, tais como: corpos materiais, sistemas ecológicos, indivíduos, comunidades e, mais recentemente, espaços geográficos (MARTIN-BREEN; ANDERIES, 2011) - de que é exemplo a resiliência regional.

Segundo a tipologia de Pendall et al. (2010), a resiliência pode ser entendida sob duas principais perspectivas: uma pressupondo a existência de um sistema de equilíbrio e outra presumindo a ocorrência de um sistema adaptativo. O pressuposto do sistema de equilíbrio é subdividido, por sua vez, em duas vertentes: uma que entende o equilíbrio como único e a outra, mais liberal, como múltiplo (CHRISTOPHERSON; MICHIE; TYLER, 2010).

A resiliência no sentido de equilíbrio único também pode ser denominada como resiliência de engenharia (SIMMIE; MARTIN, 2010). Nesse entendimento, a resiliência é expressada em termos de capacidade de resistência contra perturbações (DI CARO, 2015a) e a velocidade com que um material ou sistema retorna ao estado considerado de equilíbrio (HOLLING, 1973).

$\mathrm{Na}$ perspectiva de equilíbrio múltiplo, também denominada como resiliência ecológica (PITTERI; BRESCIANI, 2014), a resiliência é entendida como o movimento do sistema rumo a uma nova condição após a ocorrência de uma perturbação (PENDALL; FOSTER; COWELL, 2010). Entende-se que o impacto é absorvido até certo ponto e, quando o sistema se exaure, há transição para um novo ponto de equilíbrio (SIMMIE; MARTIN, 2010). Essa perspectiva preconiza a resiliência como uma capacidade de recuperação (MARTIN, 2012) e enfoca a magnitude do choque que pode ser absorvido antes que o sistema altere sua forma, função ou posição (MARTIN-BREEN; ANDERIES, 2011).

A resiliência na concepção de sistema adaptativo ou evolutivo (PIKE; DAWLEY; TOMANEY, 2010) é concebida como um atributo dinâmico que conduz um sistema a contínuos e espontâneos ajustamentos (PENDALL; FOSTER; COWELL, 2010), independentemente da ocorrência de perturbações pontuais (BATHELT; MUNRO; SPIGEL, 2013; DAVOUDI et al., 2012).

Diferentemente das outras perspectivas em que o enfoque é dado sobre a questão de ser ou não ser resiliente - sob concepção de resultado, na perspectiva evolutiva a resiliência corresponde a um processo dinâmico que envolve as habilidades de antecipação, preparação e reação às mudanças - pressupondo se tratar 
de um processo (COWELL, 2013; MARTIN, 2012). O resultado desse processo dinâmico é uma estabilidade temporária do sistema, em detrimento daquela vista como estática pela perspectiva equilibrista (BATHELT; MUNRO; SPIGEL, 2013).

Sob a visão adaptativa ou evolutiva, os choques não são interpretados, necessariamente, em sentido negativo, uma vez que podem incitar oportunidades de reinvenção e, consequentemente, conduzir a uma melhor trajetória de desenvolvimento (DUSCHL, 2016; MARTIN, 2012). Equivale a dizer que oportunidades são visualizadas em meio a adversidades (BILLINGTON et al., 2017). O Vale do Silício, o qual se reinventa regularmente por meio de ondas de inovação, pode ser um exemplo de região resiliente cujas condições favoráveis para a próxima onda de inovações são criadas quando o potencial da anterior se aproxima do esgotamento (HENTON; HELD, 2013).

Se a manutenção de estruturas passadas é o enfoque da visão de equilíbrio único, uma vez que "o status quo é o ponto de referência" (Evenhuis, 2017, p. 2) - na acepção dinâmica ou evolutiva critica-se esse status quo justamente pela sua oposição aos processos de renovação regional (BATHELT; MUNRO; SPIGEL, 2013). A resiliência adaptativa ou evolutiva envolve mais que a capacidade de absorver efeitos de crises ou reposicionar o padrão de prosperidade anterior (GONÇALVES,
2017). É a capacidade de reposicionar e fortalecer o modelo de desenvolvimento precedente, superando não apenas um quadro de recessão, mas também um contexto de desenvolvimento comprovadamente ultrapassado (GONÇALVES, 2017).

Essa capacidade de reinvenção é comumente denominada na literatura estrangeira como bounce forward (DI CARO, 2015b), a qual se trata de um contraponto à concepção equilibrista também denominada de bounce back (EVENHUIS, 2017). Um dos entendimentos mais utilizados na literatura específica de resiliência regional é o de Martin e Sunley (2015). Para eles, a resiliência regional é a capacidade dual de uma economia em resistir e se recuperar de choques por meio da restauração do status quo ou migração para um novo caminho de desenvolvimento em que seus recursos físicos, humanos e ambientais sejam empregados mais produtivamente.

O Quadro 1 contém a síntese das principais características de resiliência segundo as perspectivas discutidas. As fundamentações teóricas sobre resiliência, tais como evolução do conceito de resiliência e sua definição em variadas disciplinas, podem ser encontradas, em profundidade, nos estudos de, por exemplo: Pendall, Foster e Cowell (2010); MartinBreen e Anderies (2011); Boschma (2015) e Martin e Sunley (2015).

Quadro 1 - Síntese das perspectivas de resiliência e suas principais características

\begin{tabular}{|l|l|}
\hline Perspectivas & Principais características \\
\hline $\begin{array}{l}\text { Resiliência de } \\
\text { engenharia }\end{array}$ & $\begin{array}{l}\text { Presunção de equilíbrio único (bounce back) } \\
\text { Resiliência concebida como resistência e elasticidade de um sistema. } \\
\text { Não problematiza os processos de reorganização e reorientação }\end{array}$ \\
\hline $\begin{array}{l}\text { Resiliência } \\
\text { ecológica }\end{array}$ & $\begin{array}{l}\text { Presunção de equilíbrio múltiplo } \\
\text { Resiliência concebida como reposição e recuperação de um sistema } \\
\text { A identidade do sistema permanece a mesma }\end{array}$ \\
\hline Resiliência & $\begin{array}{l}\text { Presunção de constantes mudanças (bounce forward) } \\
\text { Resiliência concebida como reorientação ou renovação de uma trajetória de crescimento. } \\
\text { adaptativa } \\
\text { Osiliência independe de choques }\end{array}$ \\
\hline
\end{tabular}

Fonte: Martin-Breen e Anderies (2011) 


\section{METODOLOGIA}

A visualização e análise de padrões e tendências na produção científica podem ser realizadas com ferramentas cientométricas, de que é exemplo o CiteSpace (Chen, 2014). Esse programa é um dos mais famosos instrumentos de mapeamento do conhecimento (LI; MA; QU, 2017) e possibilita a identificação de padrões e tendências emergentes numa dada literatura (CHEN et al., 2012). Neste estudo, foi utilizada a versão 5.6 do mencionado programa com o objetivo de identificar os principais tópicos $\mathrm{e}$ subtópicos de discussão, bem como a evolução ao longo do tempo deles por meio da rede de cocitações de resiliência regional.

O CiteSpace gera e analisa rede de referências cocitadas conforme registros bibliográficos extraídos da Web of Science (CHEN et al., 2012), a qual pertence ao Institute for Scientific Information (ISI). Em consistência com Bar-Ilan (2008), Chen et al. (2012) e Ávila et al. (2014), justifica-se a escolha dessa base: i) pela sua natureza multidisciplinar; ii) pela indexação dos periódicos mais citados nos diversos campos científicos; iii) pela acessibilidade a todas citações recebidas por um artigo, independentemente de estarem ou não indexadas na plataforma $\mathrm{WoS}$ e, não menos importante, iv) pela compatibilidade da base com o programa CiteSpace.

Quanto aos procedimentos de coleta de produção científica, utilizou-se o critério "tópico" no buscador da base $\mathrm{Web}$ of Science. A estratégia de busca deu-se pela utilização do descritor em inglês "regional resilience", bem como da utilização de aspas, para que retornassem estudos envolvendo ambos os termos usados no descritor. Não foi utilizado intervalo de anos. A busca retornou 164 documentos (artigos, anais de congresso, livros). Dentre eles, foram selecionados apenas os artigos científicos, os quais totalizavam 125 publicações. A consulta foi realizada em meados de março de 2018.
A construção da rede de cocitações, conforme recomendado por Chen (2014), deve ser realizada por meio da expansão do quantitativo inicial de artigos incluindo-se também na análise a produção acadêmica listada nas referências deles. $\mathrm{Na}$ base de dados da WoS, esse procedimento é realizado por meio da funcionalidade "relatório de citações" e, na sequência, da seleção dos "artigos que fizeram a citação sem autocitações". Ao final desses procedimentos, foram encontradas 829 publicações, as quais foram publicadas no intervalo de 1993 a 2017.

O mapeamento das áreas da rede de cocitações deu-se pela utilização da funcionalidade "label clusters with log likelihood ratio", a qual extrai frases nominais para cada um dos agrupamentos. Justifica-se a escolha desse critério dado que, segundo Chen (2014, p. 15), "ele fornece melhores resultados em termos de exclusividade e cobertura". Além de frases nominais, os agrupamentos identificados pelo algoritmo recebem uma numeração conforme a robustez deles em termos de quantidade de artigos. Aquele mais robusto recebe a menor numeração e o menos robusto, por outro lado, a maior numeração possível (CHEN et al., 2012).

Esses autores recomendam ainda a verificação de vieses nos agrupamentos formados por meio de duas métricas: modularidade e silhueta. A modularidade sinaliza o grau que uma rede pode ser repartida em agrupamentos independentes, sendo que seu valor varia no intervalo de 0 a 1. Valores dentro do intervalo de 0,4 a 0,8 são aceitáveis (LI; MA; QU, 2017). A silhueta avalia a homogeneidade dos agrupamentos formados variando de valor no intervalo de -1 a 1 . Agrupamentos com valores acima de 0,7 sugerem homogeneidade e consistência entre os elementos (LI; MA; QU, 2017).

Além da identificação das áreas e seus respectivos tópicos de discussão, realizou-se a revisão bibliométrica acerca resiliência regional, sob o enfoque econômico e de gestão, a partir da seleção 
da área de conhecimento denominada pelo WoS como business economics. Esse procedimento retornou 61 artigos teóricos e empíricos. Essa etapa do estudo objetivou verificar as métricas demográficas da produção científica teórica e empírica levantada. Em adição, com base nos 50 artigos empíricos, objetivou-se verificar as métricas de tipificação metodológica dos artigos, bem como os fatores comumente associados por eles com a resiliência de regiões. Para o alcance desses objetivos, realizou-se análise de conteúdo pela leitura integral dos artigos com auxílio do programa NVivo 11. A Figura 1 resume as etapas e procedimentos adotados.

Figura 1 - Síntese dos procedimentos adotados de seleção de artigos a partir das funcionalidades da base WoS e da leitura dos artigos

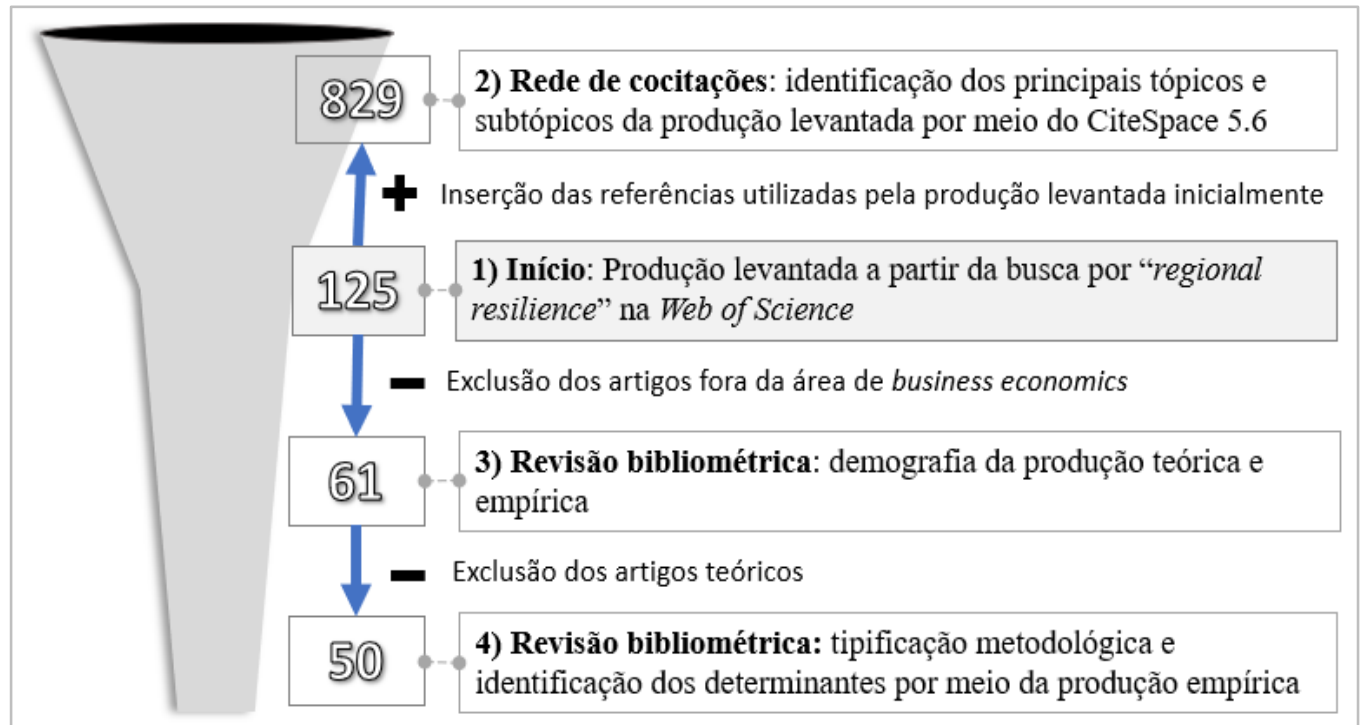

Fonte: elaboração própria

\section{PRINCIPAIS SUBTÓPICOS ACADÊMICA RESILIÊNCIA REGIONAL

$\begin{array}{rrr}\text { TÓPICOS } & \text { E } \\ \text { DA PRODUÇÃO } & \text { ACERCA }\end{array}$

O CiteSpace ilustra uma dada literatura por meio de uma rede sintetizada a partir de uma série de redes individuais. Assim, a integração dessas redes individuais possibilita uma visão geral de como um campo científico evoluiu ao longo do tempo (CHEN et al., 2012), segundo o tipo de nó escolhido. No presente estudo, utilizou-se o critério reference.

$\mathrm{O}$ valor da modularidade da rede principal foi de $\mathrm{Q}=0,74$. Ao total, foram identificados 59 agrupamentos nela. Contudo, apenas quatro apresentaram valores aceitáveis de silhueta (acima de 0,7), com base em Li, Ma e Qu (2017). Na Figura 2 são ilustrados aqueles mais robustos, conforme análise do CiteSpace. O mais robusto deles, em termos de quantidade de itens, foi o community resilience com 76 artigos. 
Figura 2 - Mapa da rede de cocitações da produção acadêmica sobre resiliência regional indexada na WoS

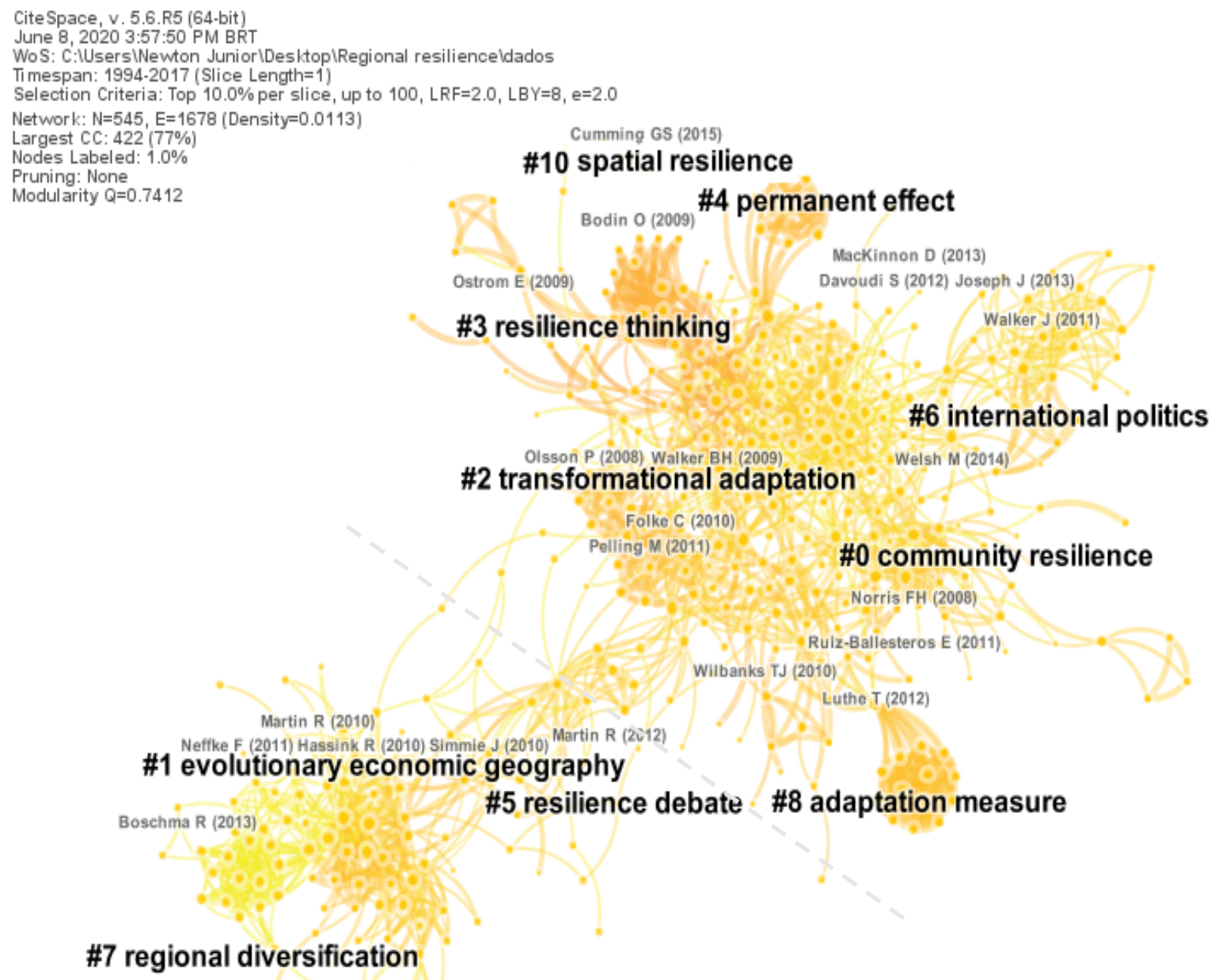

\#7 regional diversification

Fonte: CiteSpace v. 5.6

Nota-se a ramificação da rede de cocitações em duas regiões separadas pela linha tracejada (inserida pelos autores). Uma no canto superior direito envolvendo a grande maioria dos agrupamentos - os quais são baseados, sobremodo, na concepção de sistemas socioecológicos. E a outra na parte inferior esquerda abrangendo uma menor parte de aglomerados - os quais se assentam, sobremodo, na disciplina da geografia econômica evolutiva. A Tabela 1 apresenta, além dos principais tópicos de discussão, a quantidade de artigos, o ano médio das publicações pertencentes a cada tópico, os principais subtópicos e os autores mais centrais de cada um deles. 
Tabela 1 - Principais tópicos, métricas, subtópicos e autores centrais dos agrupamentos da rede de cocitações

\begin{tabular}{|l|c|c|l|c|}
\hline $\begin{array}{c}\text { Principais tópicos } \\
\text { de discussão }\end{array}$ & $\begin{array}{c}\text { Qte de } \\
\text { artigos }\end{array}$ & $\begin{array}{c}\text { Ano } \\
\text { médio }\end{array}$ & \multicolumn{1}{|c|}{$\begin{array}{c}\text { Principais subtópicos } \\
\text { de discussão do agrupamento }\end{array}$} & $\begin{array}{c}\text { Autores centrais do } \\
\text { agrupamento }\end{array}$ \\
\hline $\begin{array}{l}\text { \#0 Resiliência de } \\
\text { comunidade }\end{array}$ & 76 & 2013 & $\begin{array}{l}\text { Sistema Socioeconômico Regional / Agenda Global } \\
\text { de Sustentabilidade / Método Estatístico Analítico / } \\
\text { Regressão Linear Múltipla / Estudos de Riscos } \\
\text { Naturais/ Desastre de Inundação / Katrina / Mudança } \\
\text { Ambiental Global }\end{array}$ & $\begin{array}{c}\text { Welsh M (2014), } \\
\text { Norris F (2008), } \\
\text { Berkes F (2013) }\end{array}$ \\
\hline $\begin{array}{l}\text { \#1 Geografia } \\
\text { econômica } \\
\text { evolutiva }\end{array}$ & 59 & 2010 & $\begin{array}{l}\text { Região Industrial / Indicador Econômico / } \\
\text { Características baseadas no local / Estrutura } \\
\text { Econômica / Nova Trajetória Industrial Regional / } \\
\text { Medição da Resiliência / Análise Quantitativa / } \\
\text { Crescimento Regional do Emprego / Desempenho } \\
\text { Econômico / Diversificação econômica }\end{array}$ & $\begin{array}{c}\text { Hassink R (2010), } \\
\text { Bartin R (2010), }\end{array}$ \\
\hline $\begin{array}{l}\text { \#2 Adaptaçãona R (2015) } \\
\text { transformacional }\end{array}$ & 55 & 2011 & $\begin{array}{l}\text { Governo Local / Conceito Sociológico / Novo } \\
\text { Contrato Social / Formulador de Políticas Urbanas / } \\
\text { / Serviço Ecossistêmico Essencial Teoria da } \\
\text { Estruturação / Comportamento Espacial }\end{array}$ & $\begin{array}{c}\text { Pelling M (2011), } \\
\text { Wilbanks T (2010), } \\
\text { Walker B (2006) }\end{array}$ \\
\hline $\begin{array}{l}\text { \#3 Pensamento de } \\
\text { resiliência }\end{array}$ & 40 & 2009 & $\begin{array}{l}\text { Dualidade Ator-Estrutura / Nível de Educação / } \\
\text { Desafio de Governança / Nível de Bairro / Pesquisa } \\
\text { Aplicada / Distrito da Cidade / Conceito Sociológico } \\
\text { / Interação Socioeconômica }\end{array}$ & $\begin{array}{c}\text { Walker B (2004), } \\
\text { Folke C (2010), } \\
\text { Folke C (2006) }\end{array}$ \\
\hline
\end{tabular}

Fonte: elaboração própria a partir de informações extraídas do CiteSpace v. 5.6

No intuito de visualizar o desenvolvimento dos mencionados tópicos de discussão ao longo do tempo, recorreuse à funcionalidade timeline view do CiteSpace (Figura 3). O tamanho das circunferências representa a existência e o volume de produção acadêmica vinculado a determinado tópico em dado ano. A cor laranja representa ocorrência de uma explosão de citações associada a uma publicação específica, denominada de burst no CiteSpace.

Isso significa que "a publicação evidentemente atraiu um grau extraordinário de atenção de sua comunidade científica" (CHEN, 2014, p. 19). No presente estudo, notou-se que um dos fatores que pode estar relacionado ao destaque destes artigos em seus respectivos agrupamentos é a abordagem teórica deles, a qual oferecem conceitos e pressupostos teóricos para embasamento de estudos empíricos.

O agrupamento intitulado como "resiliência de comunidade" foi o mais robusto, em termos de quantidade de artigos. O artigo de Norris et al. (2008) apresentou o maior valor de burst $(4,03)$. Conforme definição desses autores, a resiliência de comunidade é o processo que liga um conjunto de capacidades adaptativas (recursos com atributos dinâmicos) em rede a uma trajetória positiva de funcionamento e adaptação em populações após um distúrbio (NORRIS et al., 2008). Exemplos de desastres dessa vertente: ataques terroristas de 2001, tsunami no sudeste asiático em 2004 e o furacão Katrina em 2005.

$\mathrm{O}$ artigo com maior burst $(8,71)$ do agrupamento sob o título "geografia econômica evolutiva" foi o de Martin e Sunley (2006). A abordagem da geografia econômica sobre resiliência baseia-se, especialmente, em conceitos teóricos como o de "dependência de caminho" e "lock-in" (MARTIN; SUNLEY, 2006). Infere-se do estudo que a resiliência é uma expressão da capacidade de economias regionais se reinventarem por meio de novos caminhos após choques. Esses, por sua vez, são desencadeados por forças endógenas que limitam a duração de uma condição - como, por exemplo, a adoção de uma dada tecnologia ou produção de um mesmo produto por diversas empresas (MARTIN; SUNLEY, 2006). 
Figura 3 - Ilustração do desenvolvimento dos agrupamentos ao longo do tempo da rede de cocitações

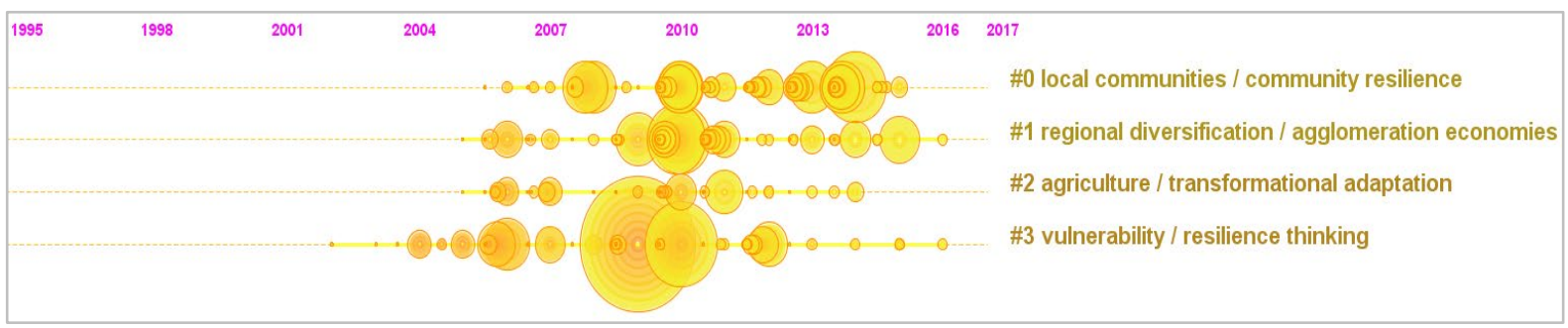

Fonte: CiteSpace v. 5.6

O agrupamento "adaptação transformacional" apresentou os artigos de Gallopín (2006) e de Smit e Wandel (2006) com o maior burst $(4,13)$. Ambos foram publicados pelo mesmo periódico e adotam uma perspectiva sistêmica. Segundo Smit e Wandel (2006), resiliência é a capacidade do sistema de lidar, adaptar ou recuperar-se dos efeitos de condições perigosas - como mudanças climáticas, por exemplo. Para Gallopín (2006), resiliência é uma mudança de estado entre os diferentes domínios de atração.

O agrupamento "pensamento de resiliência" apresentou a maior abrangência temporal, bem como a maior explosão de citações $(10,84)$ - atribuída ao artigo de Folke (2006). De acordo com esse autor, a perspectiva da resiliência é cada vez mais usada como uma abordagem para entender a dinâmica dos sistemas socioecológicos. $\mathrm{O}$ estudo narra o desenvolvimento do conceito de resiliência desde suas raízes na Ecologia, em que modelos matemáticas eram usados para compreensão de mudanças ecossistêmicas, até as ramificações nas Ciências Sociais e Ambientais. Na próxima seção, revisa-se a resiliência regional com o escopo delimitado à área de pesquisa denominada pela WoS como Business Economics, a qual englobou 61 artigos teóricos e empíricos.

\section{REVISÃO BIBLIOMÉTRICA ACERCA RESILIÊNCIA REGIONAL SOB O ENFOQUE ECONÔMICO E DE GESTÃO}

A revisão bibliométrica consiste em mensurar índices de produção e disseminação do conhecimento científico por meio do uso de técnicas quantitativas e estatísticas com o intuito de avaliar objetivamente uma determinada área ou assunto específico (ARAÚJO, 2006). Nesta etapa, o enfoque deu-se sobre a área de conhecimento denominada pela WoS como business economics, a qual apresentou 61 artigos.

A maioria da produção (72\%) acerca resiliência regional, sob a perspectiva da mencionada área, fez referência aos efeitos da crise financeira mundial de 2008/2009, corroborando Raco e Street (2012). Segundo esses autores, essa crise ressignificou o conceito de resiliência no contexto regional. Em relação ao tipo de pesquisa, a maioria dos artigos caracterizou-se como empírico (82\%) e os demais como teóricos (18\%). A distribuição dos artigos por anos revela que inicialmente, no ano de 2010, houve equilíbrio entre esses tipos de pesquisa (Figura 4). 
Figura 4 - distribuição temporal da produção acadêmica levantada

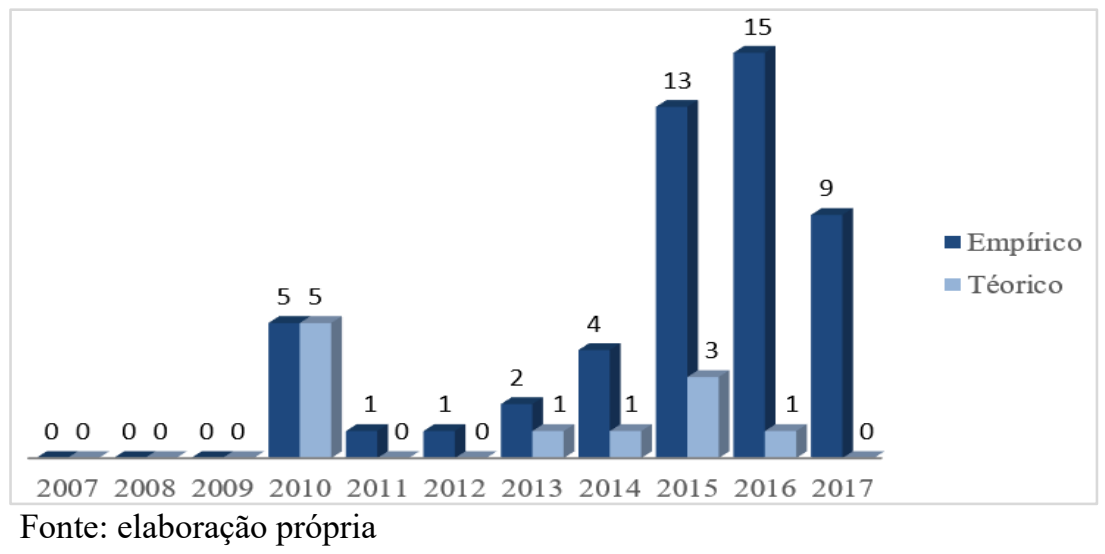

É importante ressaltar que a maior parte das publicações do ano de 2010 e 2015 ocorreu devido à publicação de duas edições especiais lançadas pelo periódico Cambridge Journal of Regions Economy and Society (CJRES) voltadas ao tema da resiliência regional. Embora em menor quantidade, os artigos eminentemente teóricos lideraram a quantidade de citações recebidas. Dos artigos teóricos publicados em 2010, apenas um não apareceu na lista dos cinco com maiores quantidades de citações recebidas, conforme a Tabela 2 . Frisa-se que as métricas apresentadas foram extraídos da WoS em consulta realizada em março de 2018.

Tabela 2 - Os artigos da produção acadêmica levantada com maiores quantidades de citações recebidas

\begin{tabular}{|l|c|l|c|}
\hline \multicolumn{1}{|c|}{ Artigos } & $\begin{array}{c}\text { Tipo de } \\
\text { pesquisa }\end{array}$ & \multicolumn{1}{|c|}{ Autoria } & $\begin{array}{c}\text { Citações } \\
\text { recebidas }\end{array}$ \\
\hline $\begin{array}{l}\text { The economic resilience of regions: towards an } \\
\text { evolutionary approach }\end{array}$ & $\begin{array}{l}\text { Téorico- } \\
\text { Empírico }\end{array}$ & Simmie e Martin (2010) & 279 \\
\hline Resilience, adaptation and adaptability & Teórico & Pike et al. (2010) & 190 \\
\hline $\begin{array}{l}\text { Regional resilience: theoretical and empirical } \\
\text { perspectives }\end{array}$ & Teórico & $\begin{array}{l}\text { Christopherson, Michie e } \\
\text { Tyler (2010) }\end{array}$ & 181 \\
\hline $\begin{array}{l}\text { Regional resilience: a promising concept to explain } \\
\text { differences in regional economic adaptability? }\end{array}$ & Teórico & Hassink (2010) & 133 \\
\hline $\begin{array}{l}\text { Resilience and regions: building understanding of } \\
\text { the metaphor }\end{array}$ & Teórico & $\begin{array}{l}\text { Pendall, Foster e Cowell } \\
(2010)\end{array}$ & 133 \\
\hline
\end{tabular}

Fonte: elaboração própria a partir de informações da WoS

A área de conhecimento da Economia, Econometria e Finanças apresentou a maior quantidade de artigos da amostra (60\%), seguida pela Geografia, Planejamento e Desenvolvimento (30\%) embora ambas tenham reunido a mesma quantidade de periódicos - e pela Administração, Gestão e Contabilidade (10\%). Nota-se a grande presença de periódicos inseridos no que poderia se chamar de interseção entre a Economia e a Geografia, a exemplo do periódico Journal of Economic Geography. Na Figura 5 estão listados os periódicos de cada área do conhecimento, assim como a expressividade em percentual de cada um deles em termos de artigos na amostra total e as respectivas métricas de fator de impacto (JIF) de cada um deles (relativas ao ano de 2017). 
Figura 5 - Distribuição da produção acadêmica levantada por área de conhecimento e por periódicos

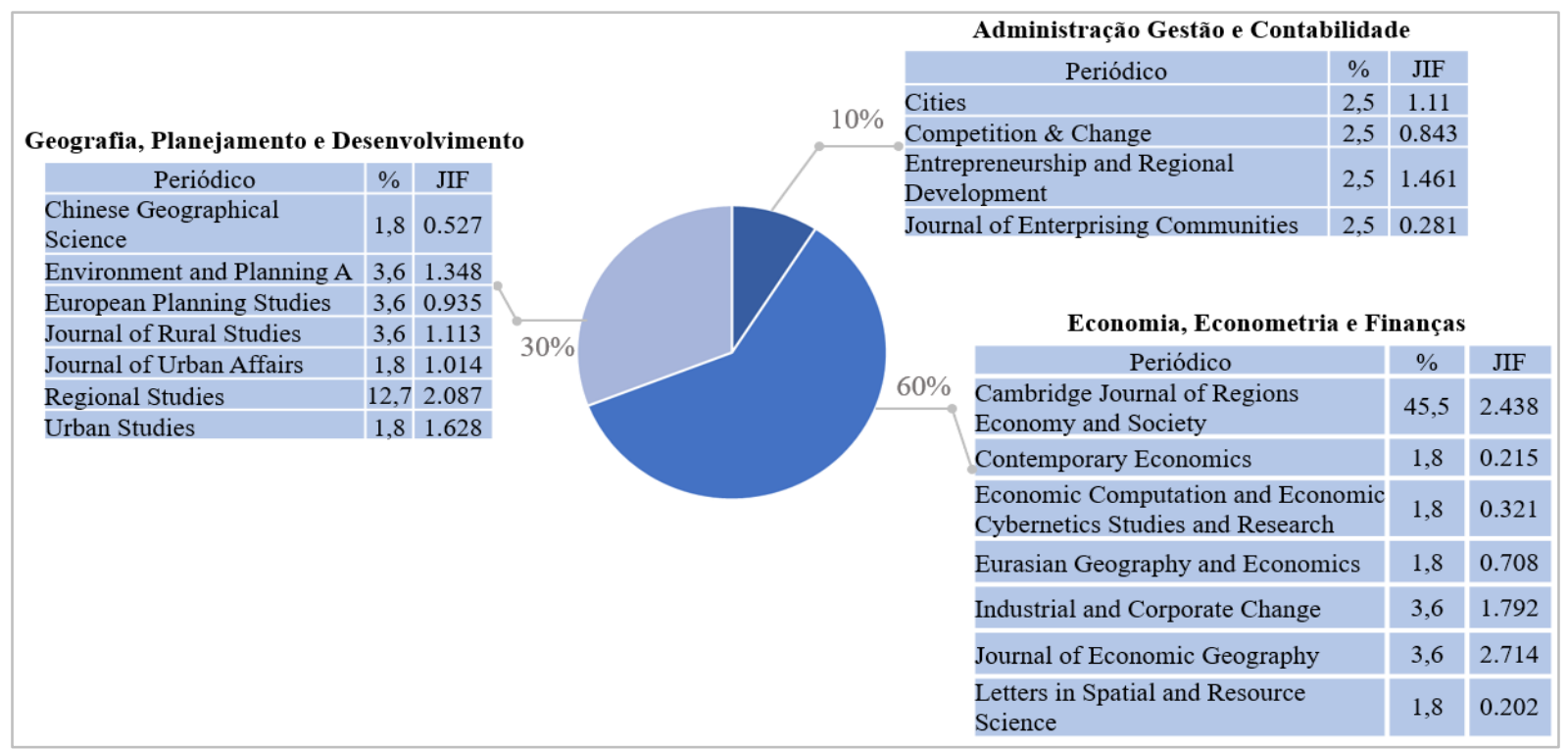

Fonte: Elaboração própria

Houve grande concentração de publicações $(45,5 \%)$ no periódico inglês Cambridge Journal of Regions Economy and Society (CJRES). Esse dado permite inferir que o debate acerca resiliência regional, sob a perspectiva da área investigada, foi especialmente promovido na última década por tal periódico. Uma evidência disso é a grande quantidade de citações recebidas por ele, a qual representou $80 \%$ do total de todas as citações recebidas pelos periódicos listados na Figura 5 - conforme dados extraídos da WoS no início de 2018.

A distribuição entre autores demonstrou-se bastante heterogênea. Ron Boschma (Universidade de Utrecht, Holanda), Gillian Bristow (Universidade de Cardiff, Reino Unido) e Ron Martin (Universidade de Cambridge, Reino Unido) destacaram-se com 3 publicações cada. Os autores que apresentaram 2 publicações foram: Juan Cuadrado-Roura (Universidad de Alcalá, Espanha), Ayda Eraydin (Universidade Técnica do Oriente Médio, Turquia), Ugo Fratesi (Politécnico de Milão, Itália), Robert Hassink (Universidade de Kiel, Alemanha), T. William Lester (Universidade da Carolina do Norte, EUA) e Andres Rodriguez-Pose
(Escola de Economia de Londres, Reino Unido). Outros 110 autores apresentaram 1 publicação.

As próximas análises baseiam-se exclusivamente nos 50 artigos empíricos para a discussão da tipificação metodológica utilizada por eles. Antes, destaca-se que, em relação aos choques, o mais mencionado nos estudos empíricos (70\%) foi a crise financeira mundial de 2008-2009. Em questões metodológicas, a abordagem de pesquisa quantitativa prevaleceu $(72 \%)$, seguida pela qualitativa (25\%) e mista (4\%). Grande parte dos artigos recorreram a dados secundários (72\%), extraídos principalmente de base de dados.

Quanto aos estudos que utilizaram dados primários $(19 \%)$, a estratégia de coleta deu-se por meio de entrevistas e questionários, ou da combinação de ambos os tipos $(9 \%)$. O recorte longitudinal dos dados $(87 \%)$ prevaleceu sobre o transversal $(13 \%)$. A abrangência temporal dos estudos apresentou mediana e média de, respectivamente, 8 e 15 anos. As maiores amplitudes temporais encontradas foram nos estudos de Cellini e Torrisi (2014) 119 anos e Lagravinese (2015) - 40 anos. 
A majoritária parte dos artigos combinou técnicas estatísticas (74\%) na etapa de análise dos dados. A análise de conteúdo foi menos frequente (19\%). O percentual residual $(7 \%)$ diz respeito a estudos que combinaram técnicas mistas de análise. Grande parte das regiões abordadas nos estudos empíricos foram do continente Europeu (60\%). A Figura 6 ilustra essa distribuição e a detalha por país. A categoria diversos refere-se a estudos comparativos entre regiões de diferentes países.

Figura 6 - Distribuição da produção levantada para a revisão bibliométrica entre continentes e

\begin{tabular}{|c|c|c|c|}
\hline Ásia & $13 \%$ & Europa & $60 \%$ \\
\hline China & 6 & Alemanha & 1 \\
\hline Japão & 1 & Croácia & 1 \\
\hline & & Espanha & 3 \\
\hline & & Grécia & 3 \\
\hline & & Holanda & 1 \\
\hline & & Itália & 6 \\
\hline & & Reino Unido & 6 \\
\hline América do $\mathrm{No}$ & $27 \%$ & Romênia & 1 \\
\hline Canadá & 5 & Suécia & 1 \\
\hline Estados Unidos & 7 & Turquia & 2 \\
\hline Diversos & 3 & Diversos & 8 \\
\hline
\end{tabular}

Fonte: Elaboração própria

Por fim, os artigos empíricos foram agrupados em categorias emergentes a partir da análise de conteúdo de seus respectivos objetivos. Para cada artigo havia a possibilidade de agrupamento em mais de uma das categorias, uma vez que elas não foram do tipo mutuamente excludentes. A Tabela 3 informa a distribuição entre elas e por anos.

Tabela 3 - Distribuição por ano e por categorias conforme objetivos dos estudos empíricos

\begin{tabular}{|l|c|c|c|c|c|c|c|c|c|}
\hline Objetivos dos estudos & 2010 & 2011 & 2012 & 2013 & 2014 & 2015 & 2016 & 2017 & $\begin{array}{c}\text { Proporção } \\
\text { relativa }\end{array}$ \\
\hline $\begin{array}{l}\text { Comparação do comportamento } \\
\text { de diferentes regiões a choque(s) }\end{array}$ & 2 & 1 & 1 & 2 & 1 & 3 & 6 & 1 & $23 \%$ \\
\hline $\begin{array}{l}\text { Proposição de modelos ou } \\
\text { conceitos para resiliência regional }\end{array}$ & 5 & 2 & 0 & 0 & 0 & 0 & 2 & 3 & $13 \%$ \\
\hline $\begin{array}{l}\text { Análise de determinantes de } \\
\text { resiliência regional }\end{array}$ & 1 & 0 & 0 & 4 & 3 & 6 & 8 & 4 & $35 \%$ \\
\hline $\begin{array}{l}\text { Análise de efeitos de choques } \\
\text { sobre regiões }\end{array}$ & 0 & 0 & 0 & 0 & 2 & 6 & 6 & 2 & $21 \%$ \\
\hline $\begin{array}{l}\text { Análise da recuperação de regiões } \\
\text { após choques }\end{array}$ & 0 & 0 & 0 & 0 & 1 & 3 & 1 & 1 & $8 \%$ \\
\hline
\end{tabular}

Fonte: Elaboração própria

A análise de determinantes de resiliência regional foi a categoria com maior proporção relativa. Ao passo que o objetivo de comparar o comportamento de diferentes regiões a choque(s) foi detectado em todos os anos, os demais concentraramse, de algum modo, em algum extremo do intervalo. A proposição de modelos ou conceitos para a resiliência regional foi mais recorrente, por exemplo, nos anos iniciais da literatura levantada. Por outro lado, os objetivos voltados à análise de determinantes de resiliência regional, assim como dos efeitos de choques sobre as 
regiões e da recuperação delas concentraram-se mais nos anos finais do intervalo. Na seção seguinte, listam-se os fatores e variáveis associados pelos estudos a determinantes de resiliência regional.

\section{OS DETERMINANTES DE RESILIÊNCIA REGIONAL SOB O ENFOQUE ECONÔMICO E DE GESTÃO}

Uma das principais questões em resiliência regional, responsável até mesmo pela popularização desse conceito (CHRISTOPHERSON; MICHIE; TYLER, 2010), diz respeito aos seus determinantes isto é, os fatores relacionados à resiliência de uma região frente aos choques (CRESCENZI; LUCA; MILIO, 2016).

Martin e Sunley (2015) propuseram um quadro-teórico composto por alguns determinantes de resiliência regional sob o viés econômico. Segundo esses autores, sob uma perspectiva de resiliência como processo, a resiliência regional é a expressão da interação de quatro principais subsistemas: estrutural e comercial; mercado de trabalho; financeiro; e governança. Entre esses subsistemas, prepondera o estrutural e comercial no debate acerca de resiliência regional, consoante Martin e Sunley (2015). Isso se deve, ainda segundo esses autores, aos esforços da geografia econômica em investigar o papel da estrutura industrial no desenvolvimento regional.

As estratégias adotadas no presente estudo para a identificação dos fatores associados a determinantes de resiliência regional pelos estudos empíricos foram: leitura integral dos artigos e o emprego da técnica da análise de conteúdo com auxílio do programa NVivo v. 11. Alguns estudos fixaram fatores de um específico fenômeno e, a partir da influência deles, estudaram a resiliência de regiões.

Exemplos são: os valores locais (HUGGINS; THOMPSON, 2015), capacidade de inovação (CLARK; HUANG; WALSH, 2010), especialização da estrutura industrial (LAGRAVINESE, 2015), empreendedorismo (BISHOP; SHILCOF, 2017), redes locais de conhecimento (CRESPO; SUIRE; VICENTE, 2016). Outros utilizaram variáveis relacionadas a variados assuntos em suas análises estatísticas. Os resultados da categorização são apresentados no Quadro 2. Também são apresentadas as referências provenientes da produção levantada.

Quadro 2 - Referências do quadro-teórico relativas às variáveis corroboradas e emergentes

\begin{tabular}{|c|c|c|c|}
\hline Categorias* & Variáveis & Subvariáveis & Referências \\
\hline \multirow{9}{*}{ 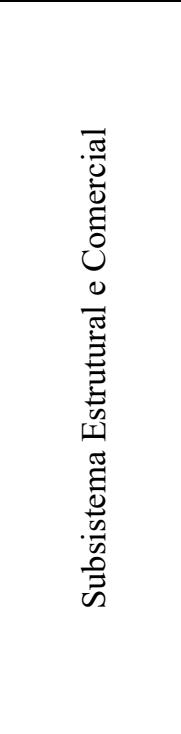 } & $\begin{array}{l}\text { Diversificação } \\
\text { econômica }\end{array}$ & $\begin{array}{l}\text { Diversificação da estrutura } \\
\text { industrial / Portfólio econômico } \\
\text { diversificado }\end{array}$ & $\begin{array}{l}\text { Wolfe (2010), Duschl (2016), Bathelt } \\
\text { et al. (2013), Hu e Hassink (2017), } \\
\text { Liang (2017), Di Caro (2015) }\end{array}$ \\
\hline & \multirow{5}{*}{$\begin{array}{l}\text { Especialização } \\
\text { econômica }\end{array}$} & Setor de serviços & Lagravinese (2015) \\
\hline & & Setor manufatureiro & $\begin{array}{l}\text { Davies (2011), Brakman et al. (2015), } \\
\text { (PALASKASY et al., 2014) Di Caro } \\
\text { (2015), Cuadrado-Roura \& Maroto } \\
(2016)\end{array}$ \\
\hline & & Setor de comércio & Petrakos e Psycharis (2016) \\
\hline & & Setor de tecnologia & $\begin{array}{l}\text { Chapple e Lester (2010), Brakman et } \\
\text { al. (2015) }\end{array}$ \\
\hline & & Setor público & Lagravinese (2015) \\
\hline & $\begin{array}{l}\text { Cadeia de } \\
\text { Suprimentos }\end{array}$ & Cadeia de suprimentos & Bristow (2010) \\
\hline & $\begin{array}{l}\text { Características das } \\
\text { empresas }\end{array}$ & Porte das empresas & $\begin{array}{l}\text { Bristow (2010), Clark et al. (2010), } \\
\text { Sabatino (2016) }\end{array}$ \\
\hline & Empreendedorismo & $\begin{array}{l}\text { Orientação empreendedora / } \\
\text { Spinoffs }\end{array}$ & Huggins e Thompson (2015) \\
\hline
\end{tabular}




\begin{tabular}{|c|c|c|c|}
\hline & Inovação & $\begin{array}{l}\text { Inovação / Abertura novas ideias } \\
\text { / Investimentos em P\&D / Base } \\
\text { industrial de média e alta } \\
\text { tecnologia / Soluções inovadoras } \\
\text { de curto-prazo }\end{array}$ & $\begin{array}{l}\text { Clark et al. (2010), Wolfe (2010), } \\
\text { Balland, Rigby e Boschma (2015), Di } \\
\text { Caro (2015), Huggins e Thompson } \\
\text { (2015), Crescenzi et al. (2016), } \\
\text { Sabatino (2016) }\end{array}$ \\
\hline & $\begin{array}{l}\text { Abertura a } \\
\text { exportações }\end{array}$ & $\begin{array}{l}\text { Abertura de mercado / } \\
\text { Internacionalização }\end{array}$ & $\begin{array}{l}\text { Petrakos e Psycharis (2016), Sabatino } \\
\text { (2016) }\end{array}$ \\
\hline \multirow{4}{*}{ 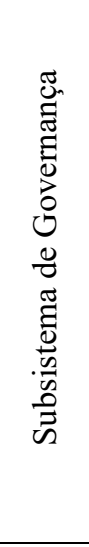 } & $\begin{array}{l}\text { Suporte } \\
\text { institucional }\end{array}$ & $\begin{array}{l}\text { Agência estatal / Ambiente } \\
\text { institucional }\end{array}$ & Eraydin (2016), Hu e Hassink (2017) \\
\hline & $\begin{array}{l}\text { Suporte de } \\
\text { instituições }\end{array}$ & $\begin{array}{l}\text { Rede entre empresas, } \\
\text { universidades, centros de } \\
\text { pesquisa }\end{array}$ & $\begin{array}{l}\text { Rutherford e Holmes (2008), Di Caro } \\
\text { (2015) }\end{array}$ \\
\hline & Cooperação & $\begin{array}{l}\text { Redes de colaboração / Redes de } \\
\text { troca de conhecimento / Relações } \\
\text { industriais / Sistema de rede / } \\
\text { Interações comerciais }\end{array}$ & $\begin{array}{l}\text { Bristow (2010), Capello, Caragliuy e } \\
\text { Fratesi (2014), Sánchez-Zamora, } \\
\text { Gallardo-Cobos, \& Ceña-Delgado } \\
\text { (2014), Di Caro (2015), Sabatino } \\
(2016)\end{array}$ \\
\hline & Ligações externas & $\begin{array}{l}\text { Redes de colaboração com atores } \\
\text { externos à região / Integração } \\
\text { com mercado global }\end{array}$ & $\begin{array}{l}\text { Bristow (2010), Capello, Caragliuy e } \\
\text { Fratesi (2014), Eraydin (2016) }\end{array}$ \\
\hline \multirow{3}{*}{ 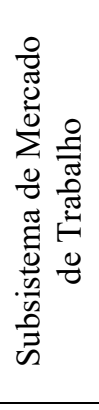 } & $\begin{array}{l}\text { Qualificação do } \\
\text { capital humano }\end{array}$ & $\begin{array}{l}\text { Nível de formação / Influência } \\
\text { do capital humano }\end{array}$ & $\begin{array}{l}\text { Di Caro (2015), Crescenzi et al. } \\
\text { (2016), Duschl (2016), Giannakis e } \\
\text { Bruggeman (2017) }\end{array}$ \\
\hline & $\begin{array}{l}\text { Flexibilidade } \\
\text { salarial e de carga } \\
\text { horária }\end{array}$ & $\begin{array}{l}\text { Reestruturação de relações } \\
\text { trabalhistas / Flexibilidade do } \\
\text { mercado de trabalho / Corte e } \\
\text { despedida de empregados }\end{array}$ & Bell e Eiser (2016) \\
\hline & $\begin{array}{l}\text { Mobilidade de } \\
\text { força de trabalho }\end{array}$ & $\begin{array}{l}\text { Mobilidade de trabalhadores / } \\
\text { Imigração de trabalhadores } \\
\text { estrangeiros }\end{array}$ & Bell e Eiser (2016) \\
\hline \multirow{2}{*}{ 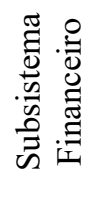 } & $\begin{array}{l}\text { Financiamentos } \\
\text { governamentais }\end{array}$ & $\begin{array}{l}\text { Concessão de empréstimos / } \\
\text { Acesso a crédito }\end{array}$ & Hu e Hassink (2017) \\
\hline & Políticas fiscais & $\begin{array}{l}\text { Austeridade fiscal / } \\
\text { Expansionismo fiscal }\end{array}$ & Bell e Eiser (2016), Davies (2011) \\
\hline
\end{tabular}

*Categorias adaptadas do modelo Martin e Sunley (2015)

Fonte: elaboração própria

\section{CONSIDERAÇÕES FINAIS}

Se inicialmente aplicada a matérias referindo-se à capacidade elástica deles de absorver $\mathrm{o}$ estresse provocado por perturbações externas, a resiliência passou a ser empregada em outras áreas do conhecimento sob novas interpretações. Os efeitos de desastres e crises nas últimas décadas, genericamente denominados de choques, têm sido abordados por meio de releitura das concepções originárias de resiliência. Conforme produção acadêmica levantada da base Web of Science, os desastres causados pelo furacão Katrina, em meados da década de 2000 e as recessões econômicas ocasionadas pela crise financeira mundial de 2008-2009 são exemplos.

Em relação à crise financeira mundial de 2008-2009, ela foi a expoente na vertente da literatura de resiliência regional formada pela área de conhecimento intitulada como business economics pela base acadêmica WoS. Denominada neste estudo como vertente econômica e de

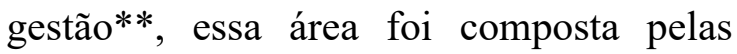
disciplinas da economia, administração e geografia. Ademais, essa vertente pode ser considerada recente, dado que seus primeiros estudos foram publicados em 2010. Não difere muito da produção ampla 
de resiliência regional considerando-se todas as áreas de conhecimento, cujos publicações datam de 1993 (um artigo) e as demais somente a partir de 2009.

O debate dentro da vertente foi conduzido majoritariamente, ao menos até o ano de 2017, por pesquisadores europeus. As regiões europeias também foram as mais abordadas, embora o choque tenha emergido nos Estados Unidos. Metodologicamente, os estudos adotaram, em maioria, a abordagem quantitativa, dados secundários, recorte longitudinal. $\mathrm{O}$ periódico inglês Cambridge Journal of Regions Economy and Society acumulou, até 2017, quase a totalidade das produções empíricas e teóricas e tem sido o líder na quantidade de citações na vertente econômica e de gestão. Esses resultados corroboram os achados de Silva e Exterckoter (2016) e Evenhuis (2017).

Embora Cowell (2013) tenha relatado a necessidade de uma consolidação dos fatores relacionados à resiliência regional e, anos depois, Cuadrado-Roura e Maroto (2016) tenham reforçado essa lacuna, não foi encontrado em busca recente - em meados de junho de 2020, estudo nesse sentido na literatura internacional indexada na WoS. Na literatura nacional, destaca-se a pesquisa bibliográfica de Silva e Exterckoter (2016) que, embora tenha contribuído à literatura de resiliência sob o enfoque de desenvolvimento regional, não se ateve à lacuna em questão.

Por outro lado, na literatura internacional, Evenhuis (2017) apresenta, sob a perspectiva evolutiva, um compilado de mecanismos por meio dos quais a adaptação e a resiliência ocorrem em vários domínios da economia regional. Além de corroborar os fatores apontados por esse autor, o presente estudo o complementa por não se ater a uma única perspectiva. Assim, entre as maiores contribuições deste estudo, destaca-se a compilação de fatores $\mathrm{e}$ variáveis presentes na vertente econômica e de gestão da literatura de resiliência regional, de 2010 a 2017, associadas à capacidade resiliente de regiões.
Os resultados encontrados também corroboram Martin e Sunley (2015) quanto à preponderância das variáveis do subsistema estrutural e comercial em relação aos demais. A importância desse compilado eleva-se, sobremodo, sob a perspectiva de que a resiliência de uma região possa ser gerenciável (FOLKE et al., 2005; MARTIN-BREEN; ANDERIES, 2011). Assim, a resiliência por constituir uma característica desejada em regiões, sobremodo na questão de desenvolvimento regional (BRISTOW, 2010) tende a, paulatinamente, ocupar maiores espaços no debate e na agenda de políticas públicas regionais (ERAYDIN, 2016).

As políticas públicas, por sua vez, devem considerar a utilização da capacidade de recursos endógenos (ERAYDIN, 2016) ou dinâmicos (NORRIS et al., 2008) de um território não apenas na recuperação de regiões, mas também na construção de estratégias para responder exitosamente a ciclos recessivos. Um imperativo adicional a essa agenda, que coloca o lugar geográfico como cerne do debate, são os novos - e assimétricos impactos econômicos trazidos pelo mais recente distúrbio mundial: a pandemia da COVID-19.

\section{REFERÊNCIAS}

ARAÚJO, C. A. Bibliometria: evolução histórica e questões atuais. Em Questão, v. 12, n. 1, p. 11-32, 2006.

ÁVILA, L. V. et al. Características Das Publicações Sobre Empreendedorismo.

Administração Pública e Gestão Social, v. 6, n. 2, p. 88-100, 2014.

BALLAND, P.-A.; RIGBY, D.; BOSCHMA, R. The technological resilience of US cities. Cambridge Journal of Regions, Economy and Society, v. 8, n. 2, p. 167-184, 2015.

BAR-ILAN, J. Which h-index? - A comparison of WoS, Scopus and Google Scholar. Scientometrics, v. 74, n. 2, p. 257-271, 2008. 
BATHELT, H.; MUNRO, A. K.; SPIGEL, B. Challenges of Transformation:

Innovation, Re-bundling and Traditional Manufacturing in Canada's Technology Triangle. Regional Studies, v. 47, n. 7, p. 1111-1130, 2013.

BELL, D. N. F.; EISER, D. Migration and fiscal policy as factors explaining the labour-market resilience of UK regions to the Great Recession. Cambridge Journal of Regions, Economy and Society, v. 9, n. 1, p. 197-215, 2016.

BILLINGTON, M. G. et al. Unfolding the relationship between resilient firms and the region. European Planning Studies, v. 25 , n. 3, p. $425-442,2017$.

BISHOP, P.; SHILCOF, D. The spatial dynamics of new firm births during an economic crisis: the case of Great Britain, 2004-2012. Entrepreneurship and Regional Development, v. 29, n. 3-4, p. 215-237, 2017.

BOSCHMA, R. Towards an Evolutionary Perspective on Regional Resilience.

Regional Studies, v. 49, n. 5, p. 733-751, 2015.

BOSCHMA, R.; MARTIN, R. The Handbook of Evolutionary Economic Geography. Cheltenham: Edward Elgar, 2010.

BRAKMAN, S.; GARRETSEN, H.; VAN MARREWIJK, C. Regional resilience across Europe: On urbanisation and the initial impact of the Great Recession.

Cambridge Journal of Regions, Economy and Society, v. 8, n. 2, p. 225240,2015

BRISTOW, G. Resilient regions: Re'place'ing regional competitiveness. Cambridge Journal of Regions, Economy and Society, v. 3, n. 1, p. 153167,2010

BROWN, D. D.; KULIG, J. C. The concept of resiliency: theoretical lessons from community research. Health and Canadian Society, v. 4, p. 29-50, 1996.
CAPELLO, R.; CARAGLIUY, A.; FRATESI, U. Spatial heterogeneity in the costs of the economic crisis in Europe: Are cities sources of regional resilience?

Journal of Economic Geography, v. 15, n. 5, p. 951-972, 2014.

CELLINI, R.; CUCCIA, T. The economic resilience of tourism industry in Italy: What the "great recession" data show. Tourism Management Perspectives, v. 16, p. 346-356, 2015.

CELLINI, R.; TORRISI, G. Regional Resilience in Italy: A Very Long-Run Analysis. Regional Studies, v. 48, n. 11, p. 1779-1796, 2014.

CHAPPLE, K.; LESTER, T. W. The resilient regional labour market? the US case. Cambridge Journal of Regions, Economy and Society, v. 3, n. 1, p. 85$104,2010$.

CHEN, C. et al. Emerging trends in regenerative medicine: a scientometric analysis in CiteSpace. Expert Opinion on Biological Therapy, v. 12, n. 5, p. 593608, 2012.

CHEN, C. The CiteSpace Manual version 0.65. [s.1.] Drexel University, 2014.

CHRISTOPHERSON, S.; MICHIE, J.; TYLER, P. Regional resilience: Theoretical and empirical perspectives. Cambridge Journal of Regions, Economy and Society, v. 3, n. 1, p. 3-10, 2010 .

CLARK, J.; HUANG, H. I.; WALSH, J. P. A typology of "innovation districts": What it means for regional resilience.

Cambridge Journal of Regions, Economy and Society, v. 3, n. 1, p. 121137, 2010.

COWELL, M. M. Bounce back or move on: Regional resilience and economic development planning. Cities, v. 30, n. 1, p. 212-222, 2013.

CRESCENZI, R.; LUCA, D.; MILIO, S. The geography of the economic crisis in 
Europe: National macroeconomic conditions, regional structural factors and short-term economic performance.

Cambridge Journal of Regions, Economy and Society, v. 9, n. 1, p. 13-32, 2016.

CRESPO, J.; SUIRE, R.; VICENTE, J.

Network structural properties for cluster long-run dynamics: Evidence from collaborative $R \& D$ networks in the European mobile phone industry.

Industrial and Corporate Change, v. 25, n. 2, p. 261-282, 2016.

CUADRADO-ROURA, J. R.; MAROTO, A. Unbalanced regional resilience to the economic crisis in Spain: A tale of specialisation and productivity.

Cambridge Journal of Regions, Economy and Society, v. 9, n. 1, p. 153$178,2016$.

DAVIES, S. Regional resilience in the 2008-2010 downturn: Comparative evidence from European countries.

Cambridge Journal of Regions, Economy and Society, v. 4, n. 3, p. 369 $382,2011$.

DAVOUDI, S. et al. Resilience: A

Bridging Concept or a Dead End?

Planning Theory and Practice, v. 13, n. 2, p. 299-333, 2012.

DI CARO, P. Recessions, recoveries and regional resilience: evidence on Italy.

Cambridge Journal of Regions, Economy and Society, v. 8, n. 2, p. 273291, 2015a.

DI CARO, P. Recessions, recoveries and regional resilience: Evidence on Italy.

Cambridge Journal of Regions, Economy and Society, v. 8, n. 2, p. 273 291, 2015b.

DUBÉ, J.; POLÈSE, M. The view from a lucky country: explaining the localised unemployment impacts of the Great Recession in Canada. Cambridge Journal of Regions, Economy and Society, v. 9, n. 1, p. 235-253, 2015.

DUSCHL, M. Firm dynamics and regional resilience: An empirical evolutionary perspective. Industrial and Corporate Change, v. 25, n. 5, p. 867-883, 2016.

ERAYDIN, A. The role of regional policies along with the external and endogenous factors in the resilience of regions. Cambridge Journal of Regions, Economy and Society, v. 9, n. 1, p. 217234, 2016.

EVENHUIS, E. New directions in researching regional economic resilience and adaptation. Geography Compass, v. 11, n. 11, p. 1-15, 2017.

FOLKE, C. et al. Adaptive Governance of Social-Ecological Systems. Annual Review of Environment and Resources, v. 30, p. 441-473, 2005.

FOLKE, C. Resilience: The emergence of a perspective for social-ecological systems analyses. Global Environmental Change, v. 16, n. 3, p. 253-267, 2006.

GALLOPÍN, G. C. Linkages between vulnerability, resilience, and adaptive capacity. Global Environmental Change, v. 16, n. 3, p. 293-303, 2006.

GIANNAKIS, E.; BRUGGEMAN, A. Determinants of regional resilience to economic crisis : a European perspective a European perspective. European Planning Studies, v. 0, n. 0, p. 1-22, 2017.

GONÇALVES, C. Regiões, cidades e comunidades resilientes : novos princípios de desenvolvimento. Revista Brasileira de Gestão Urbana, v. 9, n. 2, p. 371-385, 2017.

GORDON, J. E. Structures, or why things don't fall down. First ed. London: Penguin Books, 1978.

HENTON, D.; HELD, K. The dynamics of Silicon Valley: Creative destruction and the evolution of the innovation habitat.

Social Science Information, v. 52, n. 4, p. 539-557, 2013.

HOLLING, C. S. Resilience and

Ecological Systems. Annu.Rev.Ecol.Syst., 
v. 4, p. 1-23, 1973.

HU, X.; HASSINK, R. Exploring adaptation and adaptability in uneven economic resilience: A tale of two Chinese mining regions. Cambridge Journal of Regions, Economy and Society, v. 10, n. 3, p. 527-541, 2017.

HUGGINS, R.; THOMPSON, P. Local entrepreneurial resilience and culture: The role of social values in fostering economic recovery. Cambridge Journal of Regions, Economy and Society, v. 8, n. 2, p. 313$330,2015$.

LAGRAVINESE, R. Economic crisis and rising gaps North-South: Evidence from the Italian regions. Cambridge Journal of Regions, Economy and Society, v. 8, n. 2, p. 331-342, 2015.

LI, X.; MA, E.; QU, H. Knowledge mapping of hospitality research - A visual analysis using CiteSpace. International Journal of Hospitality Management, v. 60, p. 77-93, 2017.

LIANG, J. Trade shocks, new industry entry and industry relatedness. Regional Studies, v. 51, n. 12, p. 1749-1760, 2017.

MARTIN-BREEN, P.; ANDERIES, J. M. Resilience: A Literature Review.

Resilience: a literature review, v. 31, n. November, p. 67, 2011.

MARTIN, R. Regional economic resilience, hysteresis and recessionary shocks. Journal of Economic Geography, v. 12, n. 1, p. 1-32, 2012.

MARTIN, R.; SUNLEY, P. Path dependence and regional economic evolution. Journal of Economic Geography, v. 6, n. 4, p. 395-437, 2006.

MARTIN, R.; SUNLEY, P. On the Notion of Regional Economic Resilience: Conceptualisation and Explanation. Journal of Economic Geography, v. 15, n. 1, p. 1-50, 2015.

MASTEN, A. S.; BEST, K. M.; GARMEZY, N. Resilience and development contributions.
Developmental Psychology, v. 2, n. 1990, p. 425-444, 1990.

NORRIS, F. H. et al. Community resilience as a metaphor, theory, set of capacities, and strategy for disaster readiness. American Journal of Community Psychology, v. 41, n. 1-2, p. 127-150, 2008.

PALASKASY, T. et al. The asymmetrical impact of the economic crisis on unemployment and welfare in Greek urban economies. Journal of Economic

Geography, v. 15, n. 5, p. 973-1007, 2014.

PENDALL, R.; FOSTER, K. A.; COWELL, M. Resilience and regions: Building understanding of the metaphor. Cambridge Journal of Regions, Economy and Society, v. 3, n. 1, p. 71-84, 2010 .

PETRAKOS, G.; PSYCHARIS, Y. The spatial aspects of economic crisis in Greece. Cambridge Journal of Regions, Economy and Society, v. 9, n. 1, p. 137$152,2016$.

PIKE, A.; DAWLEY, S.; TOMANEY, J. Resilience, adaptation and adaptability. Cambridge Journal of Regions, Economy and Society, v. 3, n. 1, p. 59-70, 2010.

PITTERI, S.; BRESCIANI, L. P. Regional resilience in the theoretical and empirical perspectives: The case of the Industrial Pole of Cubatão, São Paulo . Revista Brasileira de Gestao e Desenvolvimento Regional, v. 10, n. 1, p. 305-335, 2014.

RACO, M.; STREET, E. Resilience planning, economic change and the politics of post-recession development in London and Hong Kong. Urban Studies, v. 49, n. 5, p. 1065-1087, 2012.

RUTHERFORD, T.; HOLMES, J. Engineering networks: University-industry networks in Southern Ontario automotive industry clusters. Cambridge Journal of Regions, Economy and Society, v. 1, n. 2, p. 247-264, 2008. 
SABATINO, M. Competitiveness and Resilience of the productive districts in Sicily. The behavior of the Sicilian production areas during the economic crisis. Contemporary Economics, v. 10, n. 3, p. 233-248, 2016.

SÁNCHEZ-ZAMORA, P.; GALLARDOCOBOS, R.; CEÑA-DELGADO, F. Rural areas face the economic crisis: Analyzing the determinants of successful territorial dynamics. Journal of Rural Studies, v. 35, p. 11-25, 2014.

SILVA, C. A. DA; EXTERCKOTER, R. K. Resiliência: Contribuições e Desafios para o Estudo do Desenvolvimento das Regiões. GEOgraphia, v. 18, n. 3, p. 115-
137, 2016.

SIMMIE, J.; MARTIN, R. The economic resilience of regions: Towards an evolutionary approach. Cambridge Journal of Regions, Economy and Society, v. 3, n. 1, p. 27-43, 2010.

SMIT, B.; WANDEL, J. Adaptation, adaptive capacity and vulnerability. Global Environmental Change, v. 16, n. 3, p. 282-292, 2006.

WOLFE. The strategic management of core cities: Path dependence and economic adjustment in resilient regions. Cambridge Journal of Regions, Economy and Society, v. 3, n. 1, p. 139-152, 2010.

\section{${ }^{1}$ Newton da Silva Miranda Júnior}

Doutor em Administração (UnB), Mestre em Economia (UnB), Especialista em Gestão Pública (UCDB) e Bacharel em Administração (UnB). Atualmente exerce o cargo de Administrador na Administração Pública Federal.

\footnotetext{
${ }^{2}$ Valmir Emil Hoffmann

Doutor em Administração (Universidad de Zaragoza), Mestre em Administração (UFSC), Bacharel em Ciências Econômicas (UNIVALI). Professor Titular do Departamento de Contabilidade da Universidade Federal de Santa Catarina (UFSC) com atuação nos Programas de Pós-graduação em Contabilidade (UFSC) e em Administração (PPGA/UnB).
} 